\title{
13. Modelling issue-attention dynamics in a hybrid media system
}

\author{
Annie Waldherr
}

Vasterman, Peter (ed.), From Media Hype to Twitter Storm. News Explosions and Their Impact on Issues, Crises, and Public Opinion. Amsterdam University Press, 2018

DOI: $10.5117 / 9789462982178 / \mathrm{CH} 13$

\begin{abstract}
Phenomena such as media hypes or issue-attention cycles are well studied for the traditional mass media. Today, however, traditional mass media play together with new actors in a hybrid media system. What does this imply for the dynamics of public issue attention? Should we expect more or less, higher or lower waves of attention? In this chapter, these questions are approached theoretically. Following a short review on the state of research, we will explore some extensions to an existing agent-based model of the media arena. Results show that hybridization of the media arena reduces the probability of waves of collective attention, and that specific patterns of public attention are critically influenced by the level and the diversity of attention thresholds.
\end{abstract}

Keywords: news waves, media hypes, issue attention, hybrid media system, public sphere, agent-based model

\section{Introduction}

Phenomena such as news waves (Geiß, 2011; Waldherr, 2014), media hypes (Vasterman, 2005; Wien \& Elmelund-Præstekær, 2009), or media storms (Boydstun, Hardy \& Walgrave, 2014) are well studied for the traditional mass media. Recurrent empirical patterns such as the issue-attention cycle have been identified (Brossard, Shanahan \& McComas, 2004; Downs, 1972; Luhmann, 1970; Nisbet \& Huge, 2006), and a number of self-reinforcing mechanisms have been studied that are able to explain these patterns (Waldherr, 2014). 
However, today's public sphere is undergoing fundamental structural changes. Traditional mass media are still major actors in public discourse, but play together with new media, new communication channels, and new actors in a hybrid media system (Chadwick, 2013). As there are only low thresholds for participation, public discourses on the web are potentially more diverse. They give voice to a greater number and variety of speakers, which is both welcomed for providing a high potential for participation and criticized for creating a cacophony of voices (Dahlgren, 2005; Friedland, Hove \& Rojas, 2006; Sunstein, 2001). More people than ever before can potentially participate in public communication on different scales: from personal encounters to a mass public, communication is essentially connected horizontally and vertically (Benkler, Roberts, Faris, Solow-Niederman \& Etling, 2015; Castells, 2008; Neuberger, 2009).

What do these structural changes imply for the ongoing dynamics of public attention? Do they lead to more or less, shorter or longer, higher or lower waves of attention? In this chapter, these questions are approached theoretically. First, the state of research is reviewed to describe the major structural changes of the public sphere and possible effects on public attention dynamics. Based on this review, we will explore some extensions to an agent-based model of the media arena (AMMA) that was developed in prior work (Waldherr, 2012, 2014). From the results, reasoned hypotheses are derived that may serve as guidance for future empirical studies on issue dynamics in online communication.

\section{Structural changes of the public sphere and possible consequences}

New media on the internet offer more public spaces with easier access than ever before and more personalized communication environments (Bennett \& Manheim, 2006; Papacharissi, 2002; Pariser, 2011). Unlike traditional print and broadcast media (Hilgartner \& Bosk, 1988) with its spatial restrictions and fixed publication schedules, the web's carrying capacity is virtually unrestricted. Thus, internet communication bears the potential for a higher diversity of speakers to participate in public discourse (Dahlgren, 2005; Friedland et al., 2006; Sunstein, 2001). The various public web spaces are interconnected via hyperlinks to an integrated 'networked public sphere' (Benkler, 2006; Castells, 2008; Neuberger, 2009; Van Dijk, 2006).

According to Chadwick (2011, 2013), the rise of digital media has led to a hybridization of the media system and its emerging news cycles. While 
traditional media and political elites are still powerful in controlling the news flow, non-elite actors increasingly participate in constructing news via online media. In a hybrid media environment, old and new media actors closely interact across different platforms: 'Political information cycles work on the basis of cross-platform iteration and recursion, loosening the grip of journalistic and political elites through the creation of fluid opportunity structures with greater scope for timely intervention by online citizen activists' (Chadwick, 2011: 8). This leads to a stronger influence of the public on public attention dynamics, and to a greater diversity of sources in public discourse (see also Lotan et al., 2011).

However, more participants in the public arena who are able to publish as many messages in real-time as they wish to also heavily increase competition for audience attention (Buhl, Günther \& Quandt, 2016; Chadwick, 2011). In this 'hypercompetitive environment' (Chadwick, 2011: 18) only a few prominent actors attract major attention (e.g. Adamic \& Huberman, 2000; Kwak, Lee, Park \& Moon, 2010; Pastor-Satorras \& Vespignani, 2007).

Furthermore, boundaries between private and public communication are blurring as particularly social media are also used for sharing more private information. In online networks, any information meant only for a small circle of recipients might quickly diffuse to a mass public (boyd, 2010; González-Bailón, Borge-Holthoefer \& Moreno, 2013).

In sum, the public arena appears to be more crowded, diverse, competitive, and unpredictable than ever. What do these fundamental structural changes imply for the dynamics of public attention? Do we have to expect changing patterns of public issue-attention, and, if so, in what respect? To date, there are only few studies explicitly dealing with patterns of online public attention dynamics and even less studies comparing attention dynamics both in new and traditional media.

Cacciatore et al. (2012) found different dynamics for traditional media and blogs. Their study on print and online news coverage on nanotechnology yielded that particularly blogs are likely to devote continuous attention to a certain news topic, even after its disappearance from the traditional news agenda. The authors concluded 'that the cyclical pattern of news in traditional media formats may not be a necessity online' (Cacciatore et al., 2012: 1054).

Lörcher and Neverla (2015) reported a similar observation in a study on attention dynamics towards the climate change issue in different online arenas. Their results suggested 'arena-specific attention dynamics' (Lörcher \& Neverla, 2015: 30). Online discussion arenas (e.g. lay discussion fora) showed more intense, but also more continuous issue attention over the 
course of time, whereas issue attention in mass media (e.g. online news and comments) as well as in discussion arenas induced by mass media (e.g. comments on news) was more volatile and showed higher peaks of attention.

While the results of these studies should lead us to expect less pronounced waves of attention of lower amplitudes online, studies on social media communication found different patterns. Based on five case studies, Pang (2013) proposed that social media hypes exhibit the typical anatomy of media hypes described by Wien and Elmelund-Praestekaer (2009) with one huge wave following a triggering event and two to three smaller follow-up waves.

Neuman and colleagues examined the long-term development of the jobs issue over the course of one year using big data from traditional and social media, the latter encompassing blogs and tweets. They found a basic pattern of waves of attention similar to news waves in traditional media, but issue dynamics 'are more volatile and spike more dramatically up to three times the daily average volume' (Neuman, Guggenheim, Jang \& Bae, 2014: 200).

Accordingly, in a study on diffusion patterns among twenty-eight German online news sites, Buhl, Günther, and Quandt observed abrupt surges and bursts in attention dynamics for a majority of ninety-five issues under study: 'in these cases, the timing of publication decisions among the various newsrooms in the news-site ecosystem is extraordinarily narrow' (2016:15).

As this short review shows, empirical results on issue-attention dynamics in a hybrid media system are not only sparse, but also inconsistent. While some studies found less pronounced spikes for attention dynamics in blogs and discussion fora (Cacciatore et al., 2012; Lörcher \& Neverla, 2015), others observed more volatile dynamics with abrupt and massive bursts of attention (Buhl et al., 2016; Neuman et al., 2014; Pang, 2013) in social media than in traditional media. Therefore, it seems worthwhile to further explore under which conditions we should expect which patterns with a theoretical agent-based model.

\section{The agent-based model of the media arena (AMMA)}

The approach of agent-based modelling and simulation has proven particularly valuable for theory development. The idea is to grow macroscopic patterns from the bottom up in a virtual society to find plausible explanations: 'If you didn't grow it, you didn't explain it'. (Epstein, 2006: 51) This way, agent-based computer models allow for experimenting with theoretical assumptions and help to understand what fundamental mechanisms drive the dynamics leading to empirically observed phenomena (Hedström, 2005). 
In the scope of this approach, we can explore issue-attention cycles as macro patterns dynamically arising from the interactions of societal actors in the public sphere.

In the following, a condensed description of the original agent-based model of the media arena (AMMA) as developed and reported by Waldherr $(2012,2014)$ is given. ${ }^{1}$ The model integrates the main drivers of news waves as identified by empirical research. It is not meant to mirror a specific media system or a specific issue, but to represent fundamental principles of public communication in a virtual media system. The following description is structured along the model's core assumptions for version two of the model as this model proved sufficient to generate the typical issue-attention cycle. This model version contains only journalists and neglects the role of issue sponsors who seek to influence attention dynamics by initiating (pseudo-)events.

The media arena is a public communication space with limited carrying capacity. The basic setup of the model is chosen according to the ideas of public arena models (Ferree, Gamson, Gerhards \& Rucht, 2002; Hilgartner \& Bosk, 1988). The media arena is modelled as a public space where 100 randomly distributed agents (journalists) interact with each other and with objects (three topics and a random number of events). Communication in this space is global, i.e. everything that happens can be perceived by everybody. The space is restricted in size and in the number of agents and objects it encompasses to symbolize the limited carrying capacity of the media arena. It is only two-dimensional, but wrapped to a torus, so that top and bottom as well as left and right edges are connected with each other. It represents a symbolic topic space where proximity signifies thematic similarity between topics and events, and thematic preferences of agents concerning topics and events. To keep the model simple, it contains only three topics.

Time proceeds on a discrete time scale in reporting days. In the simulation, the main procedures of the model are repeated many times in a specific order until the simulation stops. One such loop of actions is called a time step and in the AMMA represents a reporting day with the following actions: A random number of events happen and are randomly attributed to a topic. Then, the journalists move around. Each journalist chooses one interesting event, and reports it.

The higher the news value of an event, the higher is the probability of journalists reporting the event. A core assumption of news selection theory is that events have inherent characteristics making them more or less attractive to the mass media. The different news values attributed to events by journalists 
are explained by news factors such as proximity, conflict, or prominence (for a comprehensive review see Eilders, 2006). In the AMMA, events are given abstract news values indicating their reporting probability: The higher the news value of an event, the higher are its chances to be reported.

Kepplinger and Habermaier (1995) have shown that the news value of an event is not independent from the topic it is attributed to. Therefore, the AMMA also incorporates topics, which are defined as larger categories of meaning. To distinguish news values of topics from news values of events, the notions topic value and event value are used. For reasons of abstraction, one event is solely attributed to one topic in the AMMA. The arithmetic mean of event value and the respective topic value make up the news value of the event.

The more (less) journalists report a topic, the more the related topicvalue increases (decreases). The influence of topic values on news values is modelled in a positive feedback loop. According to Kepplinger's and Habermaier's (1995) theory of key events, it is assumed that topic values are not static, but develop dynamically over time. Key events gathering a lot of media attention heighten the value of associated topic categories and lead to more reports of related events. The underlying mechanism is intermedia agenda-setting (Vliegenthart \& Walgrave, 2008): Journalists observe each other and often follow the reporting choices of their colleagues.

Journalists do not report events with news values below their attention threshold for the related topic. Threshold models of public attention (ElmelundPraestekaer \& Wien, 2008; Krassa, 1988; Neuman, 1990) state that there are perception thresholds in public attention. New issues remain unnoticed until they reach a critical 'takeoff point' (Neuman, 1990: 172). In the AMMA, these thresholds are modelled as individual attention thresholds of journalists towards topics. They define the minimal required news value of events of a topic to gather the journalist's attention and be considered for reporting.

A journalist's attention threshold for a topic increases (decreases) with each day (not) reporting that topic. Collective attention towards issues tends to exhaust after a certain timespan (Downs, 1972; Luhmann, 1970; Neuman, 1990; Vasterman, 2005). Accordingly, in the AMMA, attention thresholds of journalists adapt dynamically over time: The threshold of a reported topic rises logistically while the thresholds of unreported topics fall exponentially.

Journalists prefer reporting topics they are closer to. With this assumption news bias is introduced into the model: If journalists have to select between several interesting events of high news value, they prefer the closest of these events. Communication researchers repeatedly found that individual attitudes of journalists play into the selection and framing of news (Entman, 2007; Hagen, 1993; Patterson \& Donsbach, 1996). As journalists move around 

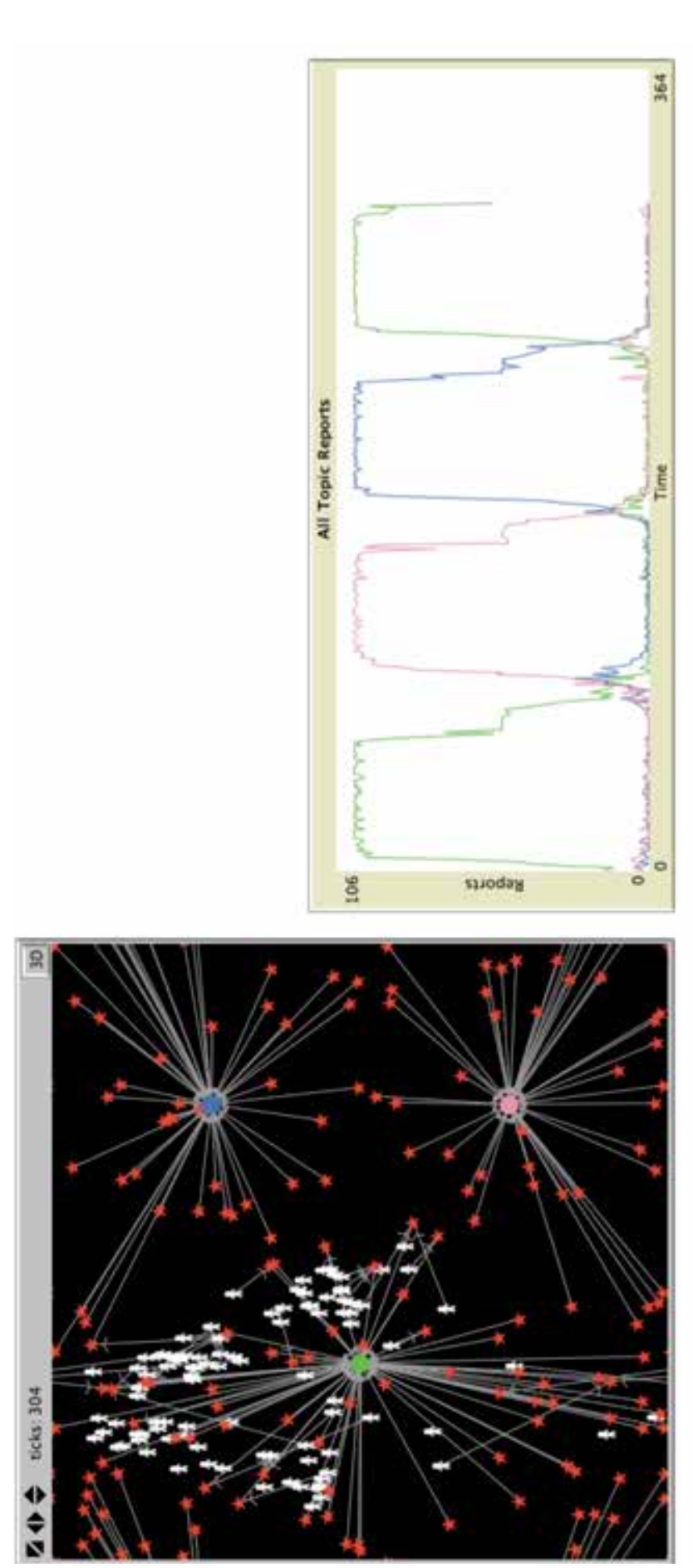

॥ ट

点

过

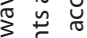

है

崩

更覀

可语要

है응

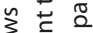

施

흥 ญ

此

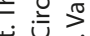

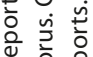

षㅎํ웡

萡

$\stackrel{5}{\circ}$

$\stackrel{\square}{\square}$

o 은

$\stackrel{n}{\underline{n}}$

० 응

บ $\frac{1}{\sqrt{0}} \frac{0}{0}$

늘 은

क जू

응

ํำ

兵方

产 $\subseteq$ 过

ษ

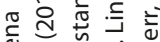

凹

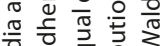

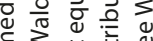

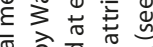

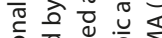

을 명 응 응

응

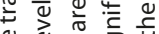

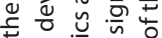

$\subseteq$ ปัํํำ

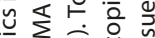

产 $\sum_{i} \dot{\overline{0}}+\frac{0}{0}$

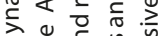

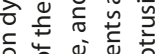

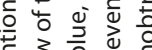

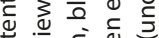

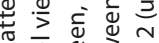

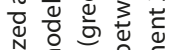

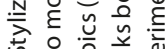

ज융웡

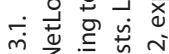

ฮัँ 
during the simulation, these topic preferences are not static, but might change over time and can be interpreted as professionalization effects of journalists in specific fields.

Journalists differ in their adoption speed of rising topics. Mathes and Pfetsch (1991) conferred the concept of opinion leadership to the media system: They found some newspapers to have a pioneering role regarding new issues and others following up later. The AMMA adopts this idea and combines it with Rogers' (2003) widely known diffusion typology, which also estimates empirical ratios for five types of innovation adopters according to a normal distribution. For reasons of abstraction, Rogers' five categories are merged to only three types of journalists: pioneers (sixteen per cent, comprising Rogers' innovators and early adopters), leaders (thirty-four per cent, early majority), and followers (fifty per cent, late majority and laggards). The three types of journalists differ in their speed as well as in the height and development of their attention thresholds. Pioneers move faster than the other journalists, and their attention thresholds are generally lower, which allows them to pick up new trends faster than all other journalists. Followers move more slowly, and their attention thresholds are generally higher so that they are the last to report on an upcoming issue. Leaders are the default type of journalists with medium speed and attention thresholds.

As has been shown in previous work (Waldherr, 2012, 2014), these assumptions are sufficient to generate issue-attention cycles of the typical pattern that have been found in numerous empirical studies (cf. Figure 13.1). In simulation experiments of the original model, heterogeneity of speakers in the media arena proved as particularly critical for generating this typical pattern Therefore, it is worthwhile to further explore issue-attention dynamics under the conditions of ever increasing heterogeneity in a hybrid media system.

\section{Extending the AMMA}

To account for the hybridization of the media arena, we add two major extensions to the model, which we explore step by step.

Step 1: Increase carrying capacity of the arena and heterogeneity of agents. We increase the number of agents in the arena to increase its carrying capacity. In their big data study of twenty-nine US issues, Neuman et al. (2014: 199) found that social media posts on specific topics outnumbered traditional media documents by a ratio of three to one. This gives us a rough empirical anchor to increase our number of agents in the media arena by 300 more agents in addition to the 100 journalists in the original AMMA. 
These agents are also designed to be more heterogeneous with respect to their main attributes. The levels of attention thresholds and speed are no longer strictly tied to specific types of journalists, but distributed randomly according to a normal distribution. ${ }^{2}$ Like Rogers (2003), we just assume that these traits are normally distributed. Apart from these changes, all agents in the model behave exactly as the journalists described above.

For reasons of abstraction, we also keep the discrete time scale of the model. However, the pace of public communication has increased dramatically with social media and online news. Thus, it is not reasonable anymore to assume that one time step equals a reporting day. It rather represents an hour or even only a couple of minutes.

Step 2: Introduce heterogeneous vision. In addition to the changes of step one, we now also vary vision among agents as another source of heterogeneity. In the original AMMA, every agent is able to perceive events in the whole arena (global vision). In this scenario, the majority of 300 agents (seventy-five per cent) are modelled as individual citizens with restricted local vision. They can only perceive and report events occurring in their neighbourhood up to a radius of four patches. At the same time, 100 (twentyfive per cent) journalists act with global vision, perceiving and potentially reporting every event that is happening in the NetLogo world. ${ }^{3}$

With this variation of vision, we account for the fact that many nonjournalistic actors in the public arena (such as bloggers or micro-bloggers) may specialize on specific topics that they report on. They may not monitor the whole topic space the way journalists of mainstream general-interest media do.

Note, however, that in this scenario we only vary vision, not outreach of agents. Topic values are still updated collectively, i.e. each report, no matter of who is counted in the collective reporting value for the topic, and this topic value partly determines the news values of related events. The news values, in turn, are modelled as objective event attributes, and not only subjective views. Thus, we assume that there is a collective understanding about what topics are newsworthy at a specific point in time. This takes into account that citizens are informed through the mass media about what the main topics at a certain point in time are. Moreover, the social web offers popularity cues such as trending topics, views, shares, and likes that allow agents to observe the choices of others.

In turn, individual agents with local vision are allowed to influence topic values with the same weight as journalistic agents with global vision. This acknowledges the fact that gatekeeping is no longer an exclusive task of journalists, but a collective effort (Friedrich, Keyling \& Brosius, 2016). For 
example, Welbers and colleagues report evidence for an increasing influence of audience metrics on journalistic news selection criteria (Welbers, Van Atteveldt, Kleinnijenhuis, Ruigrok \& Schaper, 2016). Analysing the print and online editions of five national newspapers from the Netherlands over the course of six months, they found that storylines of the most-viewed articles had higher chances to be subsequently reported.

Despite the increasing audience influence, the fundamental mechanism of gatekeeping and news selection is still working, Harcup and O'Neill (2016) conclude from an up-to-date literature review on news values in the digital age. Also, bloggers and social media users have to decide what posts to read, to like, to share, or to comment on. Often their selection criteria do not differ much from those of journalists (Eilders, Geißler, Hallermayer, Noghero \& Schnurr, 2010; Wendelin, Engelmann \& Neubarth, 2017), although there are some specific criteria that enhance 'shareability' on social media (Harcup \& O'Neill, 2016: 7), such as humour or irony (see also Büttner, 2015).

Also, the main assumptions concerning the agents' adaptive behaviour and self-reinforcing mechanisms are still appropriate according to recent studies (Büttner, 2015; Lotan et al., 2011). So far, there is no evidence suggesting that co-orientation and issue fatigue have vanished in the digital world. Furthermore, there are reasons to assume that ubiquitous popularity cues facilitate adaptive behaviour of journalists and other communicators in online public spaces.

\section{Patterns of issue attention in a hybrid media system}

In this section, the results of a series of simulation experiments with the extended versions of the AMMA are reported. As we do not have empirical information on the exact distribution of attention thresholds, we experiment with the parameters of the normal distribution. Thus, we vary mean and standard deviation of the minimal attention thresholds for both scenarios of the extended AMMA.

For the mean of minimal attention thresholds, we define three levels. The medium level of $m=0.14$ corresponds to the mean in the original AMMA, based on Rogers' (2003) assumed distribution of innovation adopters. We vary the level slightly to examine how sensitive the system reacts to a change of the level of attention thresholds. The low value of $m=0.1$ corresponds to the leader value in the original AMMA, whereas the high value of $m=0.2$ corresponds to the follower value in the original AMMA. The mean level of minimal thresholds has to be interpreted in a sense that the average 
agent never takes notice of events with a news value below the threshold. He only pays attention to events that promise a certain probability of being reported by the other agents.

The standard deviation of minimal thresholds determines how diverse the population of agents is with respect to the level of attention thresholds. We vary the standard deviation between a lower value of $s d=0.05$ to model a more homogeneous population and a higher value of $\mathrm{sd}=0.1$ to generate a more heterogeneous population.

Varying those two parameters in the described way results in six design points for each scenario of the simulation study. Figure 13.1 shows the typical patterns of public attention dynamics for the three competing issues in the model for every design point. Each coloured line indicates the simulated number of reports of an issue over the course of time. The typical patterns have been chosen from a total of ten runs for each design point to be able to check whether patterns change significantly due to stochastic influences. However, this was not the case. Instead, we find that the patterns of attention dynamics change considerably with the changes in parameters. Each combination of parameters yields a uniquely different and stable typical pattern.

Scenario 1: Increased carrying capacity and heterogeneity of agents. Concerning length and shape of the issue cycles, pattern (a) in a population with high attention thresholds and high threshold diversity corresponds most closely to the cycles of the original AMMA (cf. Figure 13.2). However, due to the increased heterogeneity of attention thresholds the waves develop more smoothly and manifest a decreased slope. Also, dynamics are more volatile in the second stage of each cycle.

This basic pattern is also stable when decreasing the level of thresholds to a medium (b) or low (c) level, but volatility rises considerably with decreased attention thresholds. As a result, the models produce longer cycles of issue attention with two (b) or even three (c) waves of attention. The pattern of design point (c) most closely resembles the stylized pattern of a social media hype proposed by Pang (2013) with three subsequent peaks after the first boom of attention in response to a triggering event.

If we also decrease threshold diversity, the basic pattern of competing issue-attention cycles is still observed for the medium (e) and low (f) threshold level. However, under these conditions the increased homogeneity of agents leads to more abrupt and steep bursts of attention, while at the same time volatility stays high. For condition (f) with both a low threshold level and low diversity attention cycles are also shorter compared to the corresponding design point (c) with high threshold diversity. 
If low threshold diversity is combined with a high threshold level (d) no pronounced waves of issue attention emerge. Over the course of the simulation, no topic is able to absorb major parts of the population of agents into the self-reinforcing dynamics. Rather all topics are reported quite constantly with random event-driven day-to-day changes and only small spikes of attention, all of them attracting less than a quarter of the population of agents.

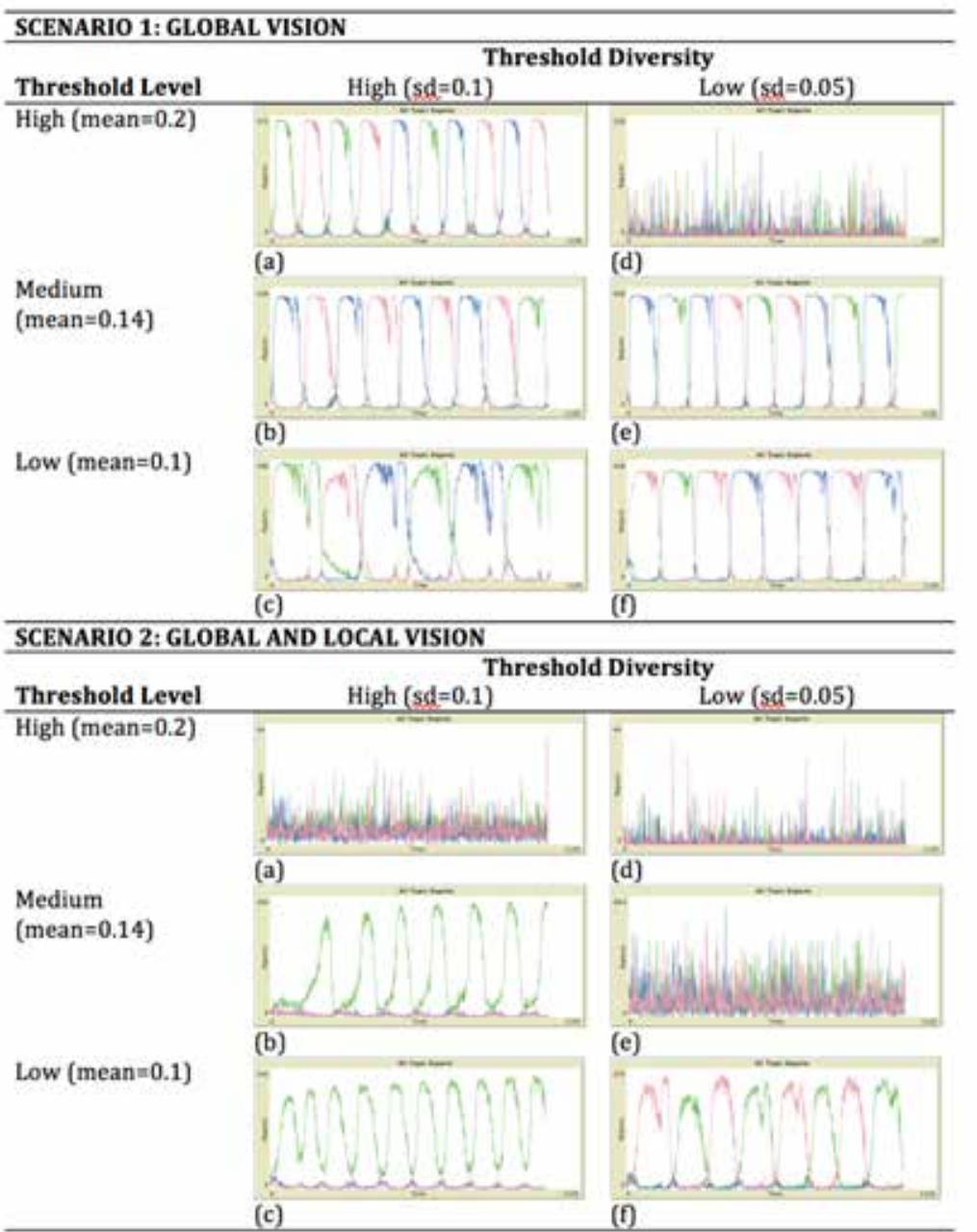

Figure 13.2. Patterns of issue attention in a hybrid media system Notes: Plots show the typical pattern of issue dynamics of three competing topics (green, blue, and red) for each experimental condition. Each simulation runs for a total of 1000 ticks. The typical patterns have been chosen from a total of ten runs for each design point. 
Scenario 2: Introduce local and global vision of agents. The alternately recurring waves of attentions for the three different topics that have been characteristic for scenario 1 are no longer observed in scenario 2 (see figure 2). Here condition (a) with high thresholds and high diversity shows a pattern of issue dynamics without waves of attention. All topics are reported with quite the same probability over the course of the simulation. Occurring spikes of attention are short and generally only attract approximately up to five per cent of the population.

When also lowering diversity of the thresholds (d), the general level of attention even decreases and shows less spikes of attention. A similar pattern is observed under condition (e) with medium threshold level and low diversity. Here, the general level of attention to the topics rises, but explicit waves do not occur either. Thus, local vision combined with high attention thresholds and low heterogeneity makes waves of shared public attention less likely to appear.

On the contrary, medium (b) to low (c) attention thresholds and high threshold diversity generates waves of issue attention again. However, waves only occur for one topic with the most advantageous starting condition, and they expose a slightly positive trend. Each new wave is of slightly higher amplitude than the preceding one. As the agents only perceive events happening within their local range of vision, only the topic with the most agents initially staying close to it has chances to profit from the self-reinforcing dynamics eventually leading to a wave.

A less extreme, but similar pattern is produced under condition (f) with low threshold level and low threshold diversity. Here, agents only hop between two topics over the course of the simulation. The waves itself are more volatile than in conditions (b) and (c), many of them showing two subsequent peaks of attention in one cycle.

For the three scenarios generating waves of collective attention under the conditions of a hybrid media system, it is worth looking into the specific patterns of journalists' and citizens' attention (see Figure 13.3). Across these three experimental design points, journalists are leading uprising issue attention in the first stage of the attention cycle. They are closely followed by citizen reports soon outperforming journalists in the number of reports. This is consistent with findings showing that the bulk of communication on blogs and in social media is follow-up communication on issues brought up by the media first (e.g. Leskovec, Backstrom \& Kleinberg, 2009; Nuernbergk, 2014).

The only experimental condition favourable to citizens leading mass media attention is a combination of low attention thresholds with high threshold diversity (c). Here, we can observe that citizen reports lead most uprisings of issue attention closely before the mass media. Under all conditions, the mass media lose interest in an issue first. This corroborates 


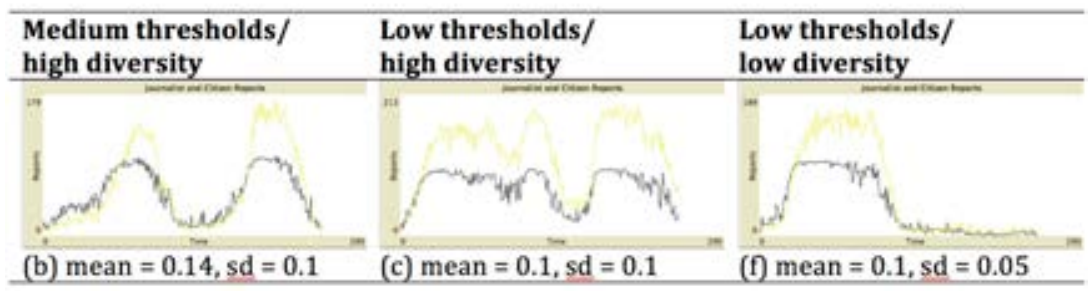

Figure 13.3. Patterns of journalists' and citizens' attention in a hybrid media system Notes: Plots show the typical pattern of issue dynamics of journalists' (black) and citizens' reports (yellow) for three experimental condition in the hybrid media scenario (2; global and local vision of agents). Each simulation runs for a total of 250 ticks. The typical patterns have been chosen from a total of ten runs for each design point.

findings of a Twitter study by Copeland, Hasell, and Bimber (2016), who empirically found a considerably longer issue-attention span of tweeting citizens than of the mass media.

\section{Discussion}

Results of the simulation study show that there is no simple answer to the question of which pattern of attention dynamics to expect in a hybrid media system. A whole spectrum of patterns emerges across the different design points of the simulation experiments. Not only the structural changes to the model matter for attention dynamics. The patterns are also critically influenced by the level and the diversity of attention thresholds, i.e. how actively users share news and how heterogeneous vs mainstreamed users are in their decisions to share.

Some generalizations may be tentatively drawn from this first qualitative exploration of the model. They are formulated as hypotheses that will need empirical investigation in the future:

H1: Increased heterogeneity of agents leads to increased volatility of issue dynamics.

H2:Lowerattention thresholds lead to increased volatility of issue dynamics.

Either one or the other condition applies to all of the scenarios of the extended AMMA. All simulated patterns expose higher volatility, but in a variety of patterns. Volatility generally rises with increasing heterogeneity of agents and lowering of attention thresholds. Both conditions are very 
characteristic of the conditions on social media platforms such as Facebook. The high number of users necessarily increases heterogeneity of actors participating in public and semi-public discussion, and the ease of sharing messages and popularity cues such as likes with friends effectively lowers attention thresholds for issues. This leads to more positive feedback loops of attention resulting in higher overall volatility of issue attention.

H3: Higher levels of attention thresholds lead to less volatile issue cycles, and above a certain point minimize the probability of waves occurring at all.

The occurrence of waves obviously highly depends on the activity of social media users. It will be interesting to further explore the exact tipping points in the model, where the system stops to produce waves. How sensitively users react, how eager they are to share critically influences how explosively the overall system reacts. In this respect, future research has to more deeply explore the specific affordances of social media platforms: What prerequisites do users need to communicate on a platform? What popularity cues are offered by the platform? And how do filter algorithms influence which messages get shared with whom? These specific conditions are likely to produce specific patterns of attention dynamics. In addition, there might also be cultural differences in user activity on social media in different countries, which might explain differences in empirically observed issue attention dynamics.

\section{H4: Global vision facilitates adaptive behaviour leading to recurring hypes.}

This is what we experience in the online news ecosystem (Buhl et al., 2016) or on social media such as Twitter and Facebook, which offer a number of popularity cues to users to enhance a shared understanding of what is newsworthy or worthy to be shared (Harcup \& O'Neill, 2016; Lin, Keegan, Margolin \& Lazer, 2014). Global vision allows communicators to quickly change their focus of attention and hop from one topic to the other. Especially steep bursts of attention are to be expected in quite homogeneous populations such as the online news ecosystem or Twitter publics that are dominated by journalists.

H5: Local vision combined with higher thresholds and lower diversity reduces the probability of waves.

These conditions may be characteristic of the blogosphere, where actors focus continuously on a topic and are less susceptible to the activities of 
others, such as the results of Cacciatore et al. (2012) as well as Lörcher and Neverla (2015) suggest. In its extreme specification, this condition is a representation of a fragmented public with no common issues.

\section{H6: Local vision combined with lower thresholds and higher diversity leads} to a lock-in of attention on certain topics.

This phenomenon might be described as a collective 'filter bubble' (Pariser, 2011). Local vision leads agents to stick to their favourite topics. Combined with a generally low level of attention thresholds and higher threshold diversity in the population this may lead to ever repeating waves of attention to the same topics or a restricted set of topics (such as the European refugee crisis in 2015 and 2016). This pattern may be fostered by personalized algorithms, which determine the individual user feeds of posts on social media.

In this series of simulation experiments, it could be shown that the level and diversity of attention thresholds are sensitive parameters in the model. Surprisingly, all of the observed patterns currently theorized and observed by media and communication scholars (such as increased volatility, abrupt bursts, multi-modal peaks, and fragmentation) could be generated with the same set of assumptions by only slightly varying the level and diversity of attention thresholds. More sensitivity analyses should follow in the future to identify the exact tipping points where the system experiences phase transitions from one macro-pattern to the other. Also, it has to be further examined whether other parameters such as agent speed or range of vision critically influence model behaviour.

More assumptions of the model may be adapted for future studies. An important extension would be to introduce social network ties between agents to represent the networked public sphere. This implies that agents will be only influenced by reporting decisions of their link neighbours at a single time step. In such a model, the influence of different network topologies on attention dynamics could be explored.

Future empirical research should more explicitly investigate the link between attention thresholds of communicators and resulting attention dynamics in online and social media. Also, more comparative research between specific public arenas with certain characteristics is needed. This also includes comparisons of different social media platforms as well as cultural contexts. Big data from different social media platforms in different countries could be systematically compared to simulation runs of the model to develop micro-explanations of the empirically observed macro-patterns. 


\section{Notes}

1. The model is implemented in NetLogo, an open-source modeling environment for building agent-based simulation models, based on the computer language Logo. The tool is available at: http://ccl.northwestern.edu/netlogo. The NetLogo model of the AMMA and a detailed documentation are available at: www.openabm.org/model $/ 4110$

2. Speed is normally distributed with mean $=1$ and $s d=1$. Minimum attention thresholds are normally distributed with mean $=0.1$ or 0.2 and $\mathrm{sd}=0.05$ or 0.1 depending on the settings of the simulation experiment (see figure 2).

3. Here we draw again on the 3 to 1 ratio reported by Neuman et al. (2014).

\section{References}

Adamic, Lada A. \& Bernardo A. Huberman (2000). 'Power-law distribution of the world wide web'. Science, 287 (5461): 2115.

Benkler, Yochai (2006). The wealth of networks: How social production transforms markets and freedom. New Haven, CT: Yale University Press.

Benkler, Yochai, Roberts, Hal, Faris, Robert, Solow-Niederman, Alicia \& Bruce Etling (2015). 'Social mobilization and the networked public sphere: Mapping the SOPA-PIPA debate'. Political Communication, 32 (4): 594-624.

Bennett, W. Lance \& Jarol B. Manheim (2006). 'The one-step flow of communication'. The Annals of the American Academy of Political and Social Science, 608 (1):213-232. boyd, danah (2010). 'Social network sites as networked publics: Affordances, dynamics, and implications'. In: Zizi Papacharissi (ed.), A networked self: Identity, community, and culture on social network sites, 39-58. New York: Routledge.

Boydstun, Amber E., Hardy, Anne \& Stefaan Walgrave (2014). 'Two faces of media attention: Media storm vs non-storm Coverage'. Political Communication, 31 (4):509-531.

Brossard, Dominique, Shanahan, James \& Katherine McComas (2004). 'Are issuecycles culturally constructed? A comparison of French and American coverage of global climate change'. Mass Communication and Society, 7 (3): 359-377.

Buhl, Florian, Günther, Elisabeth \& Thorsten Quandt (2016). 'Observing the dynamics of the online news ecosystem'. Journalism Studies, online first, 1-26.

Büttner, Vivian (2015). Akzidentielle Medienhypes: Entstehung, Dynamik und mediale Verbreitung. Wiesbaden: VS.

Cacciatore, Michael A., Anderson, Ashley A., Choi, Doo-Hun, Brossard, Dominique, Scheufele, Dietram A., Liang, Xuan, Ladwig, Peter J., Xenos, Michael \& Anthony Dudo (2012). 'Coverage of emerging technologies: A comparison between print and online media'. New Media \& Society, 14 (6): 1039-1059. 
Castells, Manuel (2008). 'The new public sphere: Global civil society, communication networks, and global governance'. The Annals of the American Academy of Political and Social Science, 616 (1): 78-93.

Chadwick, Andrew (2011). 'The political information cycle in a hybrid news system: The British prime minister and the 'bullygate' affair'. International Journal of Press/Politics, 16 (1): 3-29.

Chadwick, Andrew (2013). The hybrid media system: Politics and power. Oxford: Oxford University Press.

Copeland, Lauren, Hasell, Ariel \& Bruce Bimber (2016). 'Collective action frames, advocacy organizations, and protests over same-sex marriage', International Journal of Communication, 10: 3785-3807.

Dahlgren, Peter (2005). 'The internet, public spheres, and political communication: Dispersion and deliberation'. Political Communication, 22 (2): 147-162.

Downs, Anthony (1972). 'Up and down with ecology: The issue-attention cycle'. Public Interest, 28, 38-50.

Eilders, Christiane (2006). 'News factors and news decisions: Theoretical and methodological advances in Germany'. Communications, 31 (1): 5-24.

Eilders, Christiane, Geißler, Sebastian, Hallermayer, Michael, Noghero, Michael \& Jan-Mathis Schnurr (2010). 'Zivilgesellschaftliche Konstruktionen politischer Realität: Eine vergleichende Analyse zu Themen und Nachrichtenfaktoren in politischen Weblogs und professionellem Journalismus'. Medien und Kommunikationswissenschaft, 58 (1): 63-82.

Elmelund-Praestekaer, Christian \& Charlotte Wien (2008). 'What's the fuss about? The interplay of media hypes and politics'. InternationalJournal ofPress/Politics, 13 (3): 247-266.

Entman, Robert M. (2007). Framing bias: Media in the distribution of power.Journal of Communication, 57 (1): 163-173.

Epstein, Joshua M. (ed.) (2006). Generative social science: Studies in agent-based computational modeling. Princeton, NJ: Princeton University Press.

Ferree, Myra M., Gamson, William A., Gerhards, Jürgen \& Dieter Rucht (2002). Shaping abortion discourse: Democracy and the public sphere in Germany and the United States. Cambridge, MA: Cambridge University Press.

Friedland, Lewis A., Hove, Thomas \& Hernando Rojas (2006). 'The networked public sphere'. Javnost - The Public, 13 (4): 5-26.

Friedrich, Katja, Keyling, Till \& Hans-Bernd Brosius (2016). 'Gatekeeping revisited'. In: Gerhard Vowe \& Philipp Henn (eds), Political communication in the online world: Theoretical approaches and research designs, 59-72. New York: Routledge.

Geiß, Stefan (2011). 'Patterns of relationships between issues: An analysis of German prestige newspapers'. International Journal of Public Opinion Research, 23 (3): $265^{-286 .}$ 
González-Bailón, Sandra, Borge-Holthoefer, Javier \& Yamir Moreno (2013). 'Broadcasters and hidden influentials in online protest diffusion'. American Behavioral Scientist, 57 (7): 943-965.

Hagen, Lutz M. (1993). 'Opportune witnesses: An analysis of balance in the selection of sources and arguments in the leading German newspapers' coverage of the census issue'. European Journal of Communication, 8 (3): 317-343.

Harcup, Tony \& Deirdre O'Neill (2016). 'What is news? News values revisited (again)'. Journalism Studies, online first, 1-19.

Hedström, Peter (2005). Dissecting the social: On the principles of analytical sociology. Cambridge: Cambridge University Press.

Hilgartner, Stephen \& Charles L. Bosk (1988). 'The rise and fall of social problems: A public arenas model'. The American Journal of Sociology, 94 (1):53-78.

Kepplinger, Hans M. \& Johanna Habermeier (1995). 'The impact of key events on the presentation of reality'. European Journal of Communication, 10 (3):371-39o.

Krassa, Michael A. (1988). 'Social groups, selective perception, and behavioral contagion in public opinion'. Social Networks, 10 (2): 109-136.

Kwak, Haewoon, Lee, Changhyun, Park, Hosung \& Sue Moon (2010). What is Twitter, a social network or a news media? Paper presented at the 19th International Conference on World Wide Web, Raleigh, NC.

Leskovec, Jure, Backstrom, Lars \& Jon Kleinberg (2009). Meme-tracking and the dynamics of the news cycle. Paper presented at the 15th ACM SIGKDD International Conference on Knowledge Discovery and Data Mining, Paris, France.

Lin, Yu-Ru, Keegan, Brian, Margolin, Drew \& David Lazer (2014). 'Rising tides or rising stars? Dynamics of shared attention on Twitter during media events'. PLoS ONE, 9 (5): e94093.

Lörcher, Ines \& Irene Neverla (2015). 'The dynamics of issue attention in online communication on climate change'. Media and Communication, 3 (1): 17-33.

Lotan, Gilad, Graeff, Erhardt, Ananny, Mike, Gaffney, Devin, Pearce, Ian \& Danah Boyd (2011). 'The revolutions were tweeted: Information flows during the 2011 Tunisian and Egyptian revolutions'. International Journal of Communication, 5:1375-1405.

Luhmann, Niklas (1970). 'Öffentliche Meinung'. Politische Vierteljahresschrift, 11 (1): 2-28.

Mathes, Rainer \& Barbara Pfetsch (1991). 'The role of the alternative press in the agenda-building process: Spill-over effects and media opinion leadership'. European Journal of Communication, 6 (1): 33-62.

Neuberger, Christoph (2009). 'Internet, Journalismus und Öffentlichkeit. Analyse des Medienumbruchs'. In: Christoph Neuberger, Christian Nuernbergk, Melanie Rischke (eds), Journalismus im Internet, 19-105. Wiesbaden: VS Verlag für Sozialwissenschaften. 
Neuman, W. Russell (1990). 'The threshold of public attention'. Public Opinion Quarterly, 54: 159-176.

Neuman, W. Russell, Guggenheim, Lauren, Mo Jang, S. \& Soo Y. Bae (2014). 'The dynamics of public attention: Agenda-setting theory meets big data'.Journal of Communication, 64 (2): 193-214.

Nisbet, Matthew C. \& Mike Huge (2006). 'Attention cycles and frames in the plant biotechnology debate: Managing power and participation through the press/ policy connection'. International Journal of Press/Politics, 11 (2): 3-40.

Nuernbergk, Christian (2014). 'Follow-up communication in the blogosphere'. Digital Journalism, 2 (3): 434-445.

Pang, Augustine (2013). 'Social media hype in times of crises: Nature, characteristics and impact on organizations'. Asia Pacific Media Educator, 23 (2): 309-336.

Papacharissi, Zizi (2002). 'The virtual sphere: The internet as a public sphere'. New Media \& Society, 4 (1): 9-27.

Pariser, Eli (2011). The filter bubble: What the Internet is hiding from you. New York: Penguin Books.

Pastor-Satorras, Romualdo \& Alessandro Vespignani (2007). Evolution and structure of the Internet: A statistical physics approach. New York: Cambridge University Press.

Patterson, Thomas E. \& Wolfgang Donsbach (1996). 'News decisions: Journalists as partisan actors'. Political Communication, 13 (4): 455-468.

Rogers, Everett M. (2003). Diffusion of innovations. New York: Free Press.

Sunstein, Cass R. (2001). Republic.com. Princeton, NJ: Princeton University Press.

Van Dijk, Jan (2006). The network society: Social aspects of new media. Thousand Oaks, CA: Sage.

Vasterman, Peter (2005). 'Media-hype: Self-reinforcing news waves, journalistic standards and the construction of social problems'. European Journal of Communication, 20 (4): 508-530.

Vliegenthart, Rens \& Stefaan Walgrave (2008). 'The contingency of intermedia agenda setting: A longitudinal study in Belgium'. Journalism \& Mass Communication Quarterly, 85 (4): 860-877.

Waldherr, Annie (2012). Die Dynamik der Medienaufmerksamkeit: Ein Simulationsmodell. Baden-Baden: Nomos.

Waldherr, Annie (2014). 'Emergence of news waves: A social simulation approach'. Journal of Communication, 64 (5): 852-873.

Welbers, Kasper, Van Atteveldt, Wouter, Kleinnijenhuis, Jan, Ruigrok, Nel \& Joep Schaper (2015). 'News selection criteria in the digital age: Professional norms versus online audience metrics'. Journalism, 17 (8): 1037-1053.

Wendelin, Manuel, Engelmann, Ines \& Julia Neubarth (2017). 'User rankings and journalistic news selection'. Journalism Studies, 18 (2): 135-153. 
Wien, Charlotte \& Christian Elmelund-Præstekær, (2009). 'An anatomy of media hypes: Developing a model for the dynamics and structure of intense media coverage of single issues'. European Journal of Communication, 24 (2): 183-201.

\section{About the author}

Annie Waldherr is Assistant Professor for digitized public spheres at the University of Münster. In 2011, she received her PhD for her work on the dynamics of media attention from Freie Universität Berlin, where she was also research associate from 2010 to 2016. Before, she researched and taught at the University of Hohenheim. Her major research interests are in political communication, online communication, dynamics of the public sphere, and computational social science. Her work is published in international journals such as the Journal of Communication, Social Science Computer Review, and the Journal of Artificial Societies and Social Simulation. 
